\title{
Infecciones parasitarias intestinales y factores asociados a la infección por coccidias en pacientes adultos de un hospital público de Lima, Perú
}

\author{
Omayra Chincha L., Antonio Bernabé-Ortiz, Frine Samalvides C., \\ Leslie Soto A., Eduardo Gotuzzo H. y Angélica Terashima I.
}

\begin{abstract}
Universidad Peruana Cayetano Heredia, Lima, Perú Instituto de Medicina Tropical Alexander von Humboldt $(\mathrm{OCHL}$, FSC, LSA, EGH) Maestría de Control de Enfermedades Tropicales e Infecciosas (OCHL) Facultad de Salud Pública y Administración, Unidad de Epidemiología, ETS y SIDA (ABO)

Hospital Nacional Cayetano Heredia, Lima, Perú Departamento de Enfermedades Infecciosas, Tropicales y Dermatológicas (FSC, LSA, EGH, ATI) Laboratorio de Parasitología (ATI)
\end{abstract}

Recibido: 24 de julio de 2008 Aceptado: 17 de abril de 2009

Correspondencia a: Omayra Chincha L omychin@hotmail.com

\section{Introducción}

L as parasitosis intestinales son infecciones comunes a nivel mundial, especialmente en países con problemas de contaminación del agua y pocas condiciones de salubridad ${ }^{1}$. Aunque algunos grupos de parásitos tienen especificidad estricta, pueden afectar al humano en diversas ocasiones; por ejemplo, en pacientes con inmunosupresión se hallan parásitos como Cyclospora sp, Cryptosporidium sp, Isospora sp y Microsporidium $\mathrm{sp}^{2}$.

Se ha descrito previamente, por ejemplo, que Cryptosporidium sp es una causa común de diarrea crónica en pacientes con la infección por VIH, mientras que Cyclospora está asociada tanto a la infección por VIH como a brotes por contaminación alimentaria ${ }^{3}$. En otras ocasiones se han reportado otros parásitos causando enfermedad por la misma vía de contaminación, entre ellos Isospora sp y Microsporidium $\mathrm{sp}^{4}$.

A fines del año 2007 se estimó que en el mundo 33,2 millones de personas estaban infectados por $\mathrm{VIH}^{5}$. A nivel nacional, el Ministerio de Salud del Perú notificó 29.771 casos de infección por VIH y 20.110 casos de SIDA hasta el 31 de septiembre del $2007^{6}$. Estudios previos sobre para- sitosis intestinal en pacientes con y sin infección por VIH, encontraron que la prevalencia de estas infecciones varía según el lugar del estudio: en Brasil, 27\% de los pacientes seropositivos tuvieron parásitos intestinales en relación al 17\% de los pacientes seronegativos; mientras que, en Etiopía una proporción de $52 \%$ de pacientes infectados por VIH tuvieron infección parasitaria comparada con $42 \%$ de los pacientes ella?

En Perú, Cárcamo y cols, a través de un estudio casocontrol, encontraron que las infecciones parasitarias se presentaron en $47 \%$ de 147 pacientes con infección por VIH y que tenían diarrea por más de 7 días. En este estudio, Cryptosporidium sp fue el parásito más frecuente ( $20 \%$ los casos versus $7 \%$ los controles $)^{8}$. Otro estudio, esta vez de tipo cohorte, en pacientes infectados por VIH con diarrea, encontró factores de riesgo asociados a parasitosis como: un contacto cercano dentro del hogar, la presencia de animales como patos y pollos, compartir el mismo baño, el poco acceso al agua potable y el consumo de agua contaminada ${ }^{9,10}$. De similar forma, un estudio previo realizado en el Hospital Nacional Cayetano Heredia en pacientes con infección por VIH y diarrea crónica (mayor a dos semanas), encontró que la primera causa de parasitosis fue por Cryptosporidium sp, seguido 
por otros parásitos como Isospora belli, Giardia lamblia y Strongyloides stercoralis ${ }^{11}$.

El objetivo de este estudio fue evaluar la frecuencia de las parasitosis intestinales en pacientes con y sin infección por VIH, así como evaluar posibles factores asociados a la infección por las coccidias en pacientes atendidos en un hospital público referente de la zona norte de la ciudad de Lima.

\section{Pacientes y Métodos}

Diseño: Estudio descriptivo realizado con los reportes del Laboratorio de Parasitología del Instituto de Medicina Tropical "Alexander von Humboldt" de la Universidad Peruana Cayetano Heredia y del Departamento de Enfermedades Infecciosas, Tropicales y Dermatológicas del Hospital Nacional Cayetano Heredia, durante el año 2005.

Criterios de selección: Todos los resultados de los participantes se obtuvieron del libro de registro del Laboratorio de Parasitología. Se incluyeron los datos de pacientes que acudieron a los servicios de consulta externa y hospitalización.

El único criterio de selección usado fue tener una edad de 18 años o mayor, excluyéndose aquellos registros que no tenían datos de edad completos. No se registró la sintomatología por la cual se solicitó el estudio parasitológico, siendo una limitación del estudio. El diagnóstico de aquellos pacientes con infección por VIH fue confirmado a través de la historia que ellos mismos reportaban.

Laboratorio de Parasitología: Nuestro laboratorio es un centro docente donde se realizan las pruebas siguientes a todos los copro-parasitológicos: examen directo, técnica de Kato, técnica de sedimentación espontanea en tubo, técnica de Baermann modificada en copa por Lumbreras y técnica de sedimentación rápida de Lumbreras. Resaltaremos que se realiza la tinción de Ziehl Neelsen modificada para detectar ooquistes de coccideas, siendo por esta razón nuestro laboratorio un centro de referencia de otros centros hospitalarios.

Análisis estadísticos: Se utilizó una ficha especialmente construida para la recolección de los datos, confeccionada en base al registro de los resultados del Laboratorio de Parasitología. Se creó una base de datos en una hoja de cálculo de Microsoft Excel 2000 (Microsoft Corporartion, Washington, USA) la cual fue transferida para el análisis estadístico a SPSS 12.0 (SPSS Inc., Chicago IL, USA). Se utilizó la prueba $\chi^{2}$ o la prueba Exacta de Fisher para todas las variables categóricas. Para el cálculo de las frecuencias de infecciones parasitarias se reagruparon los datos según el status de infección por VIH; mientras que para el análisis de los factores asociados se realizó la agrupación por la presencia o ausencia de coccidias, definido como la presencia de especies de Cryptosporidium sp, Cyclospora sp e Isospora sp en los exámenes parasitológicos. Se utilizó el análisis de regresión logística para los modelos bivariados. Posteriormente, con aquellas variables que mostraron asociación, se escogió el mejor modelo multivariado de acuerdo a la prueba de likelihood ratio, tomando $\mathrm{p}<0,05$ como significativo y un intervalo de confianza (IC) al 95\%.

\section{Resultados}

Se incluyeron 2.056 pacientes que cumplieron con los criterios de selección, con una media de edad de 41,6 \pm 16,4 años (rango: 18-88), y 55,2\% varones. De acuerdo a los datos del registro, $334(16,3 \%)$ de los pacientes tenían el diagnóstico de infección por VIH, mientras que $49(2,4 \%)$ de los participantes a quienes se les hizo el análisis de parásitos estuvieron hospitalizados. En la Tabla 1 se observan las características demográficas de la población en estudio, agrupada según el diagnóstico de infección por VIH.

En la Tabla 2, observamos que Blastocystis hominis se aisló en $37,5 \%$ de los pacientes seronegativos y en $24,6 \%$ de los pacientes seropositivos $(\mathrm{p}<0,001)$, siendo el parásito más frecuente. También se detectaron Isospora belli: $2,9 \%$ en los seronegativos y $8,4 \%$ en los sero-

\begin{tabular}{|c|c|c|c|}
\hline Variables demográficas & $\begin{array}{c}\text { VIH (-)* } \\
(n=1.722)\end{array}$ & $\begin{array}{c}\text { VIH (+) } \\
(n=334)\end{array}$ & $\mathbf{p}^{* *}$ \\
\hline \multicolumn{4}{|l|}{ Sexo } \\
\hline Femenino & $788(45,8 \%)$ & $134(40,1 \%)$ & \multirow{2}{*}{0,06} \\
\hline Masculino & $934(54,2 \%)$ & $200(59,9 \%)$ & \\
\hline \multicolumn{4}{|l|}{ Edad } \\
\hline$<40$ años & $893(51,9 \%)$ & $193(57,8 \%)$ & \multirow{2}{*}{0,05} \\
\hline$\geq 40$ años & $828(48,1 \%)$ & $141(42,2 \%)$ & \\
\hline \multicolumn{4}{|l|}{ Hospitalizado } \\
\hline No & $1.692(98,3 \%)$ & $315(94,3 \%)$ & \multirow{2}{*}{$<0,001$} \\
\hline $\mathrm{Si}$ & $30 \quad(1,7 \%)$ & $19 \quad(5,7 \%)$ & \\
\hline \multicolumn{4}{|c|}{ Presencia de parásitos en el examen de heces } \\
\hline No & $933(54,2 \%)$ & $201(60,2 \%)$ & \multirow[t]{2}{*}{0,04} \\
\hline Si & $789(45,8 \%)$ & $133(39,8 \%)$ & \\
\hline
\end{tabular}


positivos ( $\mathrm{p}<0,001)$, Cryptosporidium sp: $0,4 \%$ en los seronegativos y $4,5 \%$ en los seropositivos $(p<0,001)$ y Cyclospora cayetanensis: $0,3 \%$ en los seronegativos y $3,3 \%$ en los seropositivos $(\mathrm{p}<0,001)$.

La prevalencia general de coccidias en el estudio fue de $5,4 \%(3,5 \%$ en pacientes sin infección por VIH versus
$15,0 \%$ en los pacientes con infección por VIH, $\mathrm{p}<0,001)$. En el modelo multivariado final (Tabla 3), los factores de riesgo ajustados por sexo asociados a la infección por coccidias fueron la infección por el VIH $(p<0,001)$ y estar hospitalizado ( $\mathrm{p}=0,035)$; mientras la edad $\geq 40$ años de edad fue un factor protector $(\mathrm{p}=0,007)$.
Tabla 2. Infecciones parasitarias intestinales en pacientes adultos de un hospital público de Lima, Perú. Parásitos más comunes en la población de estudio. ( $n: 2.056)$

\begin{tabular}{|c|c|c|c|c|}
\hline Parásitos hallados & $\begin{array}{c}\text { Total } \\
(\mathrm{n}=\mathbf{2 . 0 5 6})\end{array}$ & $\begin{array}{c}\text { VIH }(-) \\
(n=1.722)\end{array}$ & $\begin{array}{c}\text { VIH (+) } \\
(n=334)\end{array}$ & P* \\
\hline Blastocystis hominis & $727(35,4 \%)$ & $645(37,5 \%)$ & $82(24,6 \%)$ & $<0,001$ \\
\hline Strongyloides stercoralis & $123(6,0 \%)$ & $112(6,5 \%)$ & $11 \quad(3,3 \%)$ & 0,023 \\
\hline Isospora belli & $78 \quad(3,8 \%)$ & $50 \quad(2,9 \%)$ & $28 \quad(8,4 \%)$ & $<0,001$ \\
\hline Giardia lamblia & $77 \quad(3,8 \%)$ & $63 \quad(3,7 \%)$ & $14 \quad(4,2 \%)$ & 0,637 \\
\hline Entamoeba histolytica & $24 \quad(1,2 \%)$ & $20 \quad(1,2 \%)$ & $4 \quad(1,2 \%)$ & 1,0 \\
\hline Trichuris trichiura & $23 \quad(1,1 \%)$ & $21 \quad(1,2 \%)$ & $2(0,6 \%)$ & 0,566 \\
\hline Cryptosporidium sp & $21 \quad(1,0 \%)$ & $6 \quad(0,4 \%)$ & $15(4,5 \%)$ & $<0,001$ \\
\hline Ascaris lumbricoides & $20 \quad(1,0 \%)$ & $19(1,1 \%)$ & $1 \quad(0,3 \%)$ & 0,231 \\
\hline Cyclospora cayetanensis & $16(0,8 \%)$ & $5 \quad(0,3 \%)$ & $11 \quad(3,3 \%)$ & $<0,001$ \\
\hline Hymenolepis nana & $14(0,7 \%)$ & $10 \quad(0,6 \%)$ & $4 \quad(1,2 \%)$ & 0,263 \\
\hline
\end{tabular}

Tabla 3: Infecciones parasitarias intestinales en pacientes adultos de un hospital público de Lima, Perú. Análisis bivariado y multivariado para factores de riesgo de infección por coccidias

$\begin{array}{|lrccc|}\text { Variable } & \begin{array}{c}\text { Coccidia (-) } \\ (\mathbf{n = 1 . 9 6 1 )}\end{array} & \begin{array}{c}\text { Coccidia (+) } \\ (\mathbf{n}=\mathbf{9 5})\end{array} & \begin{array}{c}\text { Análisis } \\ \text { bivariado }\end{array} & \begin{array}{c}\text { Análisis } \\ \text { multivariado* }\end{array} \\ \text { Sexo } & 878(45,1 \%) & 44(40,0 \%) & 1 & \\ \text { Femenino } & 1.068(54,9 \%) & 66(60,0 \%) & 1,23(0,83-1,82) & \\ \text { Masculino } & & & & 1 \\ \text { Edad } & 1.012(52,0 \%) & 74(67,3 \%) & 1 & 1 \\ <40 \text { años } & 933(48,0 \%) & 36(32,7 \%) & 0,53(0,35-0,79) & 0,57(0,37-0,86) \\ \geq 40 \text { años } & & & & 1 \\ \text { Hospitalizado } & 1.905(97,9 \%) & 102(92,7 \%) & 1 & 1 \\ \text { No } & 41(2,1 \%) & 8(7,3 \%) & 3,64(1,67-7,98) & 2,42(1,06-5,52) \\ \text { Si } & & & & 1 \\ \text { Infección por VIH } & 1.662(85,4 \%) & 60(54,6 \%) & 1 & 1 \\ \text { No } & 284(15,6 \%) & 50(45,4 \%) & 4,88(3,28-7,24) & 4,53(3,03-6,77) \\ \text { Sí } & & & & \end{array}$

*Resultados ajustados por sexo.

\section{Discusión}

La frecuencia de las parasitosis intestinales encontradas en este estudio es comparable con la encontrada en estudios realizados con anterioridad en nuestro medio 9 . Los resultados demuestran que $B$. hominis es el parásito más frecuente en los pacientes atendidos en este hospital público de Lima. En general, este parásito es el más común, tanto en pacientes con infección por VIH, así como aquellos sin la infección, similar a lo indicado por otros autores ${ }^{10,11}$. Blastocystis hominis es un parásito al cual no se le reconoce aún sintomatología específica; sin embargo, algunos trabajos han correlacionado su hallazgo a diarrea (aguda o crónica), náuseas, vómitos, flatulencia, sin tener un característica propia ${ }^{12}$. Como se observa en el presente estudio, la prevalencia de parásitos que producen infección intestinal es diferente a otros trabajos. En el estudio de Mohandas ${ }^{13}$ se encontró 30\% de exámenes parasitológicos positivos en una población de pacientes seropositivos para VIH; Cryptosporidium parvum (10,8\%) fue el parásito más común, Giardia lamblia se encontró en $8,3 \%$ mientras que $B$. hominis y $C$. cayetanensis se presentaron en $3,3 \%$ cada una e $I$. belli en $2,5 \%$. La presencia de diarrea asociada al hallazgo de parásitos fue de $75 \%$. En el estudio de Senegal ${ }^{14}$, Microsporidium sp $(9,4 \%)$ fue el agente causal de diarrea en pacientes con infección por VIH, seguido de Cryptosporidium sp (8,2\%) siendo $B$. hominis causante de diarrea en $2,5 \%$ y en pacientes sin infección por VIH en $0,6 \%$. En el estudio de Prasad ${ }^{15}, I$. belli fue el agente más común como causante de diarrea (31\%), seguido de Cryptosporidium sp (11\%). En nuestro estudio $B$. hominis fue el agente más frecuente hallado en pacientes con seropositivos para VIH comparándolo con otras realidades, aunque fue más frecuente en pacientes seronegativos $(p<0,001)$. Este agente, reconocido actualmente como parásito, debe tenerse en cuenta en el diagnóstico inicial para su tratamiento oportuno, tanto en pacientes con como sin la infección por VIH, en nuestro medio. Isospora belli y Cryptosporidium sp siguen siendo las coccidias más frecuentes por lo que su búsqueda debe de realizarse en todo paciente con diagnóstico de infección por VIH que llegue a nuestros consultorios con diarrea.

Isospora belli, C. cayetanensis y Cryptosporidium $\mathrm{sp}$ son parásitos que se encuentran asociados a la infección por VIH. La disminución progresiva de las células del 
sistema inmunitario, específicamente CD4, hace al portador de la infección por VIH susceptible a la aparición de parásitos oportunistas ${ }^{14,8}$. Las coccidias son un grupo de parásitos que pueden tener alta morbi-mortalidad en seropositivos si es que los afectados no reciben un tratamiento apropiado ${ }^{15,16}$.

Después de ajustar por sexo, el modelo multivariado mostró que la infección por VIH y el estar hospitalizado fueron factores de riesgo asociados a infección por coccidias, mientras que la edad mayor de 40 años fue un factor protector. Aunque no se pudo evaluar directamente el nivel de inmunosupresión de los pacientes con infección por VIH, el hecho de estar hospitalizado puede dar una medida indirecta del nivel de compromiso inmunológico de estos, viéndose afectados por infecciones oportunistas y requiriendo hospitalización para su tratamiento ${ }^{17}$. Este hallazgo es apoyado por el hecho que 5,7\% de los pacientes con infección por VIH se encontraban hospitalizados, comparado con $1,7 \%$ de los seronegativos, siendo esta diferencia estadísticamente significativa.

La edad también fue un factor de riesgo asociado a la infección por coccidias. Aunque no es clara la relación, podría ser explicado por el efecto causado por la frecuencia de la infección en las personas que acuden al Laboratorio de Parasitología. Como se conoce, las personas jóvenes son más vulnerables a la adquisición del VIH debido mayormente al riesgo de exposición por relaciones sexuales no protegidas ${ }^{7}$. En el Perú, la mediana de la edad de todos los casos de SIDA es de 31 años, lo cual significa que la mitad de los casos se han infectado antes de los 25 años, lo cual podría explicar la asociación vista en este estudio ${ }^{6}$.

El número de casos involucrados en este estudio le da una buena potencia para detectar diversos factores asociados. Sin embargo, una limitación es que está basado sólo en registros, lo que impide un mejor análisis y reconocimiento de otros factores asociados a las parasitosis. Debido a esto, como se explicó anteriormente, no se pudo realizar la evaluación del nivel de células CD4 en los casos de los pacientes infectados por el VIH.

Algunas medidas pueden tomarse para reducir el riesgo de infección por coccidias, la mayoría de las cuales debe estar centrada en la disminución en la transmisión de la infección por el VIH, sobre todo en la población joven, considerada actualmente como población en riesgo. Si el paciente esta infectado por VIH, una adecuada profilaxis o el recibir tratamiento anti-retroviral de gran actividad podrían evitar la morbilidad y mortalidad por coccidias ${ }^{17}$. Enfatizar la profilaxis primaria en pacientes con la infección por VIH según lo indican las guías actuales (tratamiento profiláctico con un recuento de CD4 menor de 200 células $/ \mathrm{mm}^{3}$ ). El uso de sulfas ayuda a disminuir la incidencia de infecciones por Pneumocystis jiroveci, Toxoplasma gondii, I. belli, C. cayetanensis y Microsporidium sp, por lo que su utilización disminuiría el riesgo de infecciones por coccidias y otros agentes mencionados ${ }^{18}$.

Limitaciones del estudio fueron no tener la sintomatología al momento de la toma de muestra, siendo una dificultad estandarizar los datos procedentes de otros centros y la lectura del historial clínico, por lo cual el dato de ser seropositivo fue sólo a libre voluntad del paciente el comentarlo en la recolección de datos en el laboratorio. En el Perú la confidencialidad del diagnóstico de infección por VIH está protegida por ley.

\section{Resumen}

Objetivos: Evaluar la frecuencia de parásitos intestinales y factores asociados a la infección por coccidias en un hospital público de Lima-Perú. Métodos: Estudio descriptivo, transversal, realizado con los reportes del Laboratorio de Parasitología. Se incluyeron pacientes de $>18$ años, ambulatorios u hospitalizados, que tuvieron al menos una muestra de heces evaluada. Usando regresión logística, se calcularon OR e IC al 95\%. Resultados: Se incluyó 2.056 pacientes en el análisis; 55,2\% fueron varones y $334(16,3 \%)$ fueron seropositivos para VIH. La infección parasitaria más frecuente fue Blastocystis hominis $(35,4 \%)$. El modelo multivariado ajustado por sexo mostró que la infección por VIH $(\mathrm{OR}=4,53)$, estar hospitalizado $(\mathrm{OR}=2,42)$ y la edad $\geq 40$ años $(\mathrm{OR}=$ $0,57)$ estuvieron asociados con infección por coccidias. Conclusiones: Blastocystis hominis se aisló frecuentemente en pacientes con y sin infección por VIH. Estar hospitalizado y ser seropositvo para VIH fueron factores de riesgo para infección por coccidias, mientras la edad $\geq 40$ años fue un factor protector.

\section{Referencias}

1.- Okhuysen P C, White A C Jr. Parasitic infections of the intestine. Curr Opin Infect Dis 1999; 12 (5): 467-72.

2.- Fantry Lori. Gastrointestinal infections in the immunocompromised host. Curr Opin Gastroenterol 2005; 21 (1): 39-43.
3.- Christie J, Garcia L. Emerging parasitic infections. Clin Lab Med 2004; 12 : 737-72.

4.- Simao Ferreira, M. Infections by protozoa in inmunocompromised hosts. Mem Inst Oswaldo Cruz 2000, 95: 159-62.

5.- Situación de la epidemia de SIDA. Informe Especial sobre la prevención del VIH < en línea>, diciembre 2007. ONUSIDA-OMS. URL disponible en versión español: www.unaids.org.

6.- Ministerio de Salud. Epidemiología del SIDA en el Perú: Boletín Mensual y Vigilancia Epidemiológica del VIH/SIDA en el Perú, < en línea > septiembre del 2007, URL disponible en: http:// www.oge.sld.pe/vigilancia/vih/Boletin_2007/ noviembre.pdf 
7.- Lewthwaite P, Gill G, Hart A, Beeching N. Gastrointestinal parasites in the immunocompromised. Curr Opin Infect Dis 2005; 18 : 427-35.

8.- Carcamo C, Hooton T, Wener M, Weiss N, Gillman R, Arévalo J, et al. Etiologies and manifestations of persistent diarrhea in adults with HIV-1 infections: A case-control study in Lima, Perú. J Infect Dis 2005; 191: 11-9.

9.- Bern C, Kawai V, Vargas D, Rabke-Verami J, Williamson J, Chavez-Valdez R, et al. The epidemiology of intestinal microsporidiosis in patients with HIV/AIDS in Lima-Peru. J Infect Dis 2005; 191: 1658-66.

10.- Leder K, Hellard M, Sinclair M, Fairley C, Wolfe R. No correlation between clinical symptoms and Blastocystis hominis in inmunocompetent individuals. J Gastroenterol Hepatol 2005; 20: $1390-4$

11.- García C, Rodríguez E, Do Natalie, López de Castilla Diego, Terashima A, Gotuzzo E. Parasitosis intestinal en pacientes con la infección
VIH-SIDA. Rev Gastroenterol Peruana 2006; 26: 21-4.

12.- Huang D B, White A C. An updated review on cryptosporidium and giardia. Gastroenterol Clin North Am 2006; 35: 291-314

13.- Mohandas, Sehgal R, Sud A, Malla N. Prevalence of intestinal parasitic pathogens in HIVseropositive individuals in Northern India. Jpn J Infect Dis 2002; 55 (3): 83-4

14.- Gassama A, Sow PS, Fall F, Camara P, GuèyeN'diaye A, Seng R, et al. Ordinary and opportunistic enteropathogens associated with diarrhea in Senegalese adults in relation to human immunodeficiency virus serostatus. Int J Infect Dis. 2001; 5 (4): 192-8.

15.- Prasad K N, Nag V L, Dhole T N, Ayyagari A. Identification of enteric pathogens in HIV-positive patients with diarrhoea in northern India. J Health Popul Nutr 2000 Jun; 18 (1): 23-6.

16.- Arenas-Pinto A, Certad G, Ferrara G, Castro J, Bello M A, Núñez L T. Association between parasitic intestinal infections and acute or chronic diarrhoea in HIV-infected patients in Caracas, Venezuela. Int J STD AIDS 2003; 14: 487-92.

17.- Centers for Disease and Prevention. Guidelines for Prevention and Treatment of Opportunistic Infections in HIV-Infected Adults and Adolescents. Recommendations from CDC, the National Institutes of Health, and the HIV Medicine Association Infectious Diseases Society of America. Treating Opportunistic Infections among HIV-Infected Adults and Adolescents. MMWR Morb Mortal Wkly Rep 2009; 58: RR4 (1-207)

18.- St. Georgiev V. Opportunistic infections-treatment and prophylaxis. Human Press 2000.

19.- Hunter PR, Nichols G. Epidemiology and clinical features of Cryptosporidium infection in immunocompromised patients. Clin Microbiol Rev 2002; 15 (1): 145-54. Review.

20.- Tello R, Canales M. Técnicas de diagnóstico de enfermedades causadas por entero-parásitos. Revista Diagnóstico 2000; 39 (4): Julio-Agosto. 